\title{
Nonohmic conductivity as a probe of crossover from diffusion to hopping in two dimensions
}

\author{
G. M. Minkov, A. A. Sherstobitov, O. E. Rut, and A. V. Germanenko \\ Institute of Physics and Applied Mathematics, Ural State University, 620083 Ekaterinburg, Russia
}

(Dated: October 31, 2018)

\begin{abstract}
We show that the study of conductivity nonlinearity gives a possibility to determine the condition when the diffusion conductivity changes to the hopping one with increasing disorder. It is experimentally shown that the conductivity of single quantum well GaAs/InGaAs/GaAs heterostructures behaves like diffusive one down to value of order $10^{-2} e^{2} / h$.

PACS numbers: 73.20.Fz, 73.61.Ey
\end{abstract}

Two opposite models are used to describe the conductivity in two dimensional (2D) systems at low temperature. The first one considers an electron as free particle with well defined quasimomentum, which sometime scatters by static scattering potential. The quantum corrections to the conductivity (due to interference and interaction) determine in the main the temperature and magnetic field dependences of the conductivity. It is clear this model is valid for $k_{F} l>1$, where $k_{F}$ and $l$ are Fermi quasimomentum and mean free path, respectively.

The second model treats electrons as localized particles which rarely hope from one localized state to another one. All the results for this case are obtained in the framework of percolation theory which is valid when the probability of the transitions between the states is very small so that the dispersion of the probabilities is exponentially large and percolation theory becomes applicable. This theory gives ${ }^{1,2}$

$$
\rho(T)=\rho_{0}(n, T) \exp \left(T_{0} / T\right)^{p}, 0<p<1 .
$$

where $n$ is electron density and $T_{0}$ is constant depending on localization length. The value of $p$ depends on relationship between $k_{B} T$ and Coulomb gap. This expression is valid when the exponent $\left(T_{0} / T\right)^{p}$ is larger than $10 \stackrel{2}{2}$ The value of $\rho_{0}(n, T)$ depends on overlapping of the wave functions of the localized states and hopping mechanism, but for all cases it is larger than $h / e^{2}$. So, the expressions for hopping conductivity are valid when conductivity is less than $10^{-4} e^{2} / h$.

Thus the conductivity of $2 \mathrm{D}$ systems has to be described by theory of quantum corrections when $\sigma>$ $e^{2} / h$ and by theory of hoping conductivity when $\sigma<$ $10^{-4} e^{2} / h$. Over wide conductivity range $e^{2} / h>\sigma>$ $10^{-4} e^{2} / h$ both approaches are not applicable in the strike sense. However, it is commonly accepted that the conductivity mechanism in this range is hopping and practically all the experimental data are treated in framework of the theory of hopping conductivity.

One of theoretical reasoning behind this conclusion is the well known Landauer formula which shows that the conductivity of one open channel is $e^{2} / h$. For the first sight the $2 \mathrm{D}$ random network of the open $1 \mathrm{D}$ channels should have the conductivity $e^{2} / h$ also. This is true with- out taking into account interference. However, the closed paths arise in such network and the interference of the waves propagated in the different channels has to lead to decreasing of the conductivity value. Therefore it seems that the value of conductivity $e^{2} / h$ is not lower limit for the diffusive conductivity when the transport is by delocalized states.

Experimentally, the conclusion on the conductivity mechanism comes from an analysis of the temperature dependence of the conductivity. When $\sigma<e^{2} / h$, the experimental dependences $\rho(T)$ can be satisfactorily described by Eq. (11) with $0.3 \lesssim p \lesssim 0.8{ }^{3.4 .5 .6 .7 .8}$ Relaying on such value of power $p$, practically all the authors conclude that the variable range hopping regime takes place.

We emphasize that a number of unusual features is observed in such a 2D hopping regime as compared with the well-studied $3 \mathrm{D}$ variable range hopping conductivity. The are the following.

1. The less than unity value of $p$ is observed up to relatively high temperature, $T \simeq 5-10 \mathrm{~K}$. The transition to the nearest-neighbor hopping regime $\left(\epsilon_{2}\right.$-regime $)$ or to the conductivity by free carriers ( $\epsilon_{1}$-regime) is not observed usually.

2. The value of $\rho_{0}$ is close to $h / e^{2}$ practically in all cases independent of the structure type and carrier density.

3. The Hall coefficient is temperature independent, its value is determined by the electron density, $n$, down to very low conductivity values of order $10^{-2} e^{2} / h$ : $R_{H}=(e n)^{-1}$.

4. The negative magnetoresistance is observed down to $\sigma \simeq(0.1-0.01) e^{2} / h$ and its shape is very close to that observed for high conductivity $\sigma \gg e^{2} / h$, when it is caused by suppression of interference quantum correction.

Another approach to interpret the conductivity in this intermediate range $e^{2} / h>\sigma>10^{-4} e^{2} / h$ was used in Ref. 9. The authors attempted to trace the changes in electron transport with decreasing conductivity caused by increasing disorder starting from $\sigma \gg e^{2} / h$. They analyzed not only the temperature dependences of the con- 
ductivity but the magnetic field dependences of the conductivity tensor components at low and high magnetic field. A surprising conclusion has been arrived: all the transport effects are well described within the framework of the quantum correction theory down to conductivity significantly less than $e^{2} / h$.

In the present paper we demonstrate that the study of nonohmic conductivity can elucidate the low temperature conductivity mechanism when $\sigma<e^{2} / h$. An idea of experiment will be clear after consideration of the nature of conductivity nonlinearity in diffusive and hopping regimes.

Diffusive regime. At low temperature, changing of the conductivity $\sigma=j / E$ with electric field and, thus, with injected power $Q=j E$ originates in this case from electron heating. The heating leads to growth of the conductivity via lowering of the quantum corrections magnitude which is determined by electron temperature ${ }^{10}$

For steady state the injected power $Q$ is equal to the energy relaxation rate $P$ which depends on the electron and lattice temperature, $T_{e}$ and $T_{l}$, respectively. This equilibrium determines the value of $T_{e}$ for given value of $T_{l}$. When the energy relaxation rate $P\left(T_{e}, T_{l}\right)$ is governed by the interaction with phonons it is equal to the difference of two identical functions, one of them depends on the electron temperature and another one depends on the lattice temperature 12

$$
P\left(T_{e}, T_{l}\right)=F\left(T_{e}\right)-F\left(T_{l}\right) .
$$

From this equation it follows that $\partial P\left(T_{e}, T_{l}\right) / \partial T_{e}$ does not depend on $T_{l}$

$$
\frac{\partial P\left(T_{e}, T_{l}\right)}{\partial T_{e}}=\frac{\partial F\left(T_{e}\right)}{\partial T_{e}} .
$$

Experimentally, the derivative $\partial P\left(T_{e}, T_{l}\right) / \partial T_{e}$ can be straightforwardly obtained from the dependences $\sigma(T)$ measured at low injected power and $\sigma(Q)$ measured at fixed lattice temperature. Differentiating these dependences we can find the experimental value of relative nonlinearity introduced as

$$
\eta=\left.\frac{\partial \sigma(Q) / \partial Q}{\partial \sigma(T) / \partial T}\right|_{\sigma(Q)=\sigma(T)}
$$

which is exactly equal to $\left(\partial F\left(T_{e}\right) / \partial T_{e}\right)^{-1}$. Indeed,

$$
\begin{aligned}
\left.\frac{\partial \sigma(Q) / \partial Q}{\partial \sigma(T) / \partial T}\right|_{\sigma(Q)=\sigma(T)} & =\frac{\left(\partial \sigma / \partial T_{e}\right)\left(\partial T_{e} / \partial Q\right)}{\partial \sigma / \partial T} \\
& =\frac{\partial T_{e}}{\partial Q}=\left[\frac{\partial F\left(T_{e}\right)}{\partial T_{e}}\right]^{-1} .
\end{aligned}
$$

Hopping regime. First of all, the conductivity in this regime depends both on the lattice and electron temperature. Second, the changing of the conductivity with electric field results not only from the electron heating but from the increasing of the probabilities of hops and impact ionization of localized states as well. Finally, the

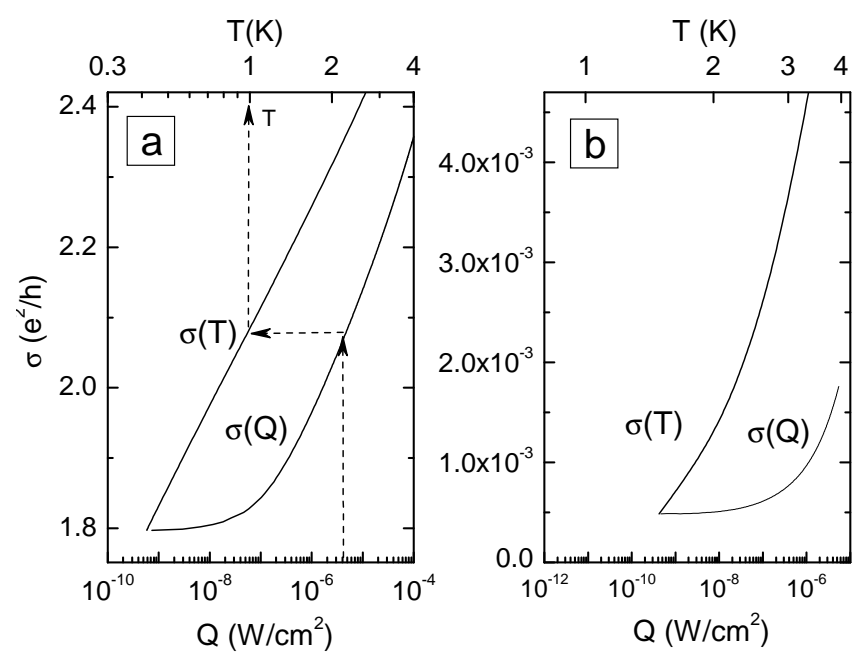

FIG. 1: Temperature and power dependences of the conductivity for $\sigma(1.5 K)=2.2 e^{2} / h$ (a) and $\sigma(1.5 K)=6 \times$ $10^{-4} e^{2} / h(\mathrm{~b})$.

energy distribution function of the electrons in electric field can deviate from the Fermi-Dirak function and the approximation of electron temperature can fail. All these effects have to lead to changing in relative nonlinearity and, thus, the value of $\eta$ will depend on both electron and lattice temperature.

Thus, we suggest a simple experimental way to distinguish the diffusive conductivity from the hopping one. As long as the conductivity remains diffusive the $T_{e}$ dependences of the relative nonlinearity $\eta$ measured at different lattice temperatures $T_{l}$ have to fall on common curve and this property has to disappear when the conductivity becomes hopping.

Experimentally, we investigated the relative nonlinearity of the conductivity for two types of the single quantum well heterostructures GaAs/InGaAs/GaAs. The first one consists of $0.5 \mathrm{mkm}$-thick undoped GaAs epilayer, a $\mathrm{Sn} \delta$-layer, a $9 \mathrm{~nm}$ spacer of undoped GaAs, a 8 $\mathrm{nm} \mathrm{In}_{0.2} \mathrm{Ga}_{0.8}$ As well, a $9 \mathrm{~nm}$ spacer of undoped GaAs, a $\mathrm{Sn} \delta$-layer, and a $200 \mathrm{~nm}$ cap layer of undoped GaAs. The electron density and mobility were $n=4 \times 10^{15} \mathrm{~m}^{-2}$ and $\mu=0.65 \mathrm{~m}^{2} /(\mathrm{V} \mathrm{s})$, respectively. The samples were mesa etched into Hall bars on which basis field-effect transistors with an $\mathrm{Ag}$ or $\mathrm{Al}$ gate electrode were fabricated. It was very important to use thick insulator between gate electrode and 2D channel to decrease the influence of voltage drop over the channel. We used the $10 \mathrm{mkm}$ organic insulator.

Heterostructures of another type were analogous except that they had no doping layers. The conductivity and electron density of such samples varied by illumination from $10^{-4}$ to $10 e^{2} / h$ and from $1 \times 10^{15}$ to $4 \times 10^{15} \mathrm{~m}^{-2}$, respectively. Results obtained for both types of the structures were close.

In what follows the results obtained for different electron density will be referenced by the value of $\sigma$ measured 

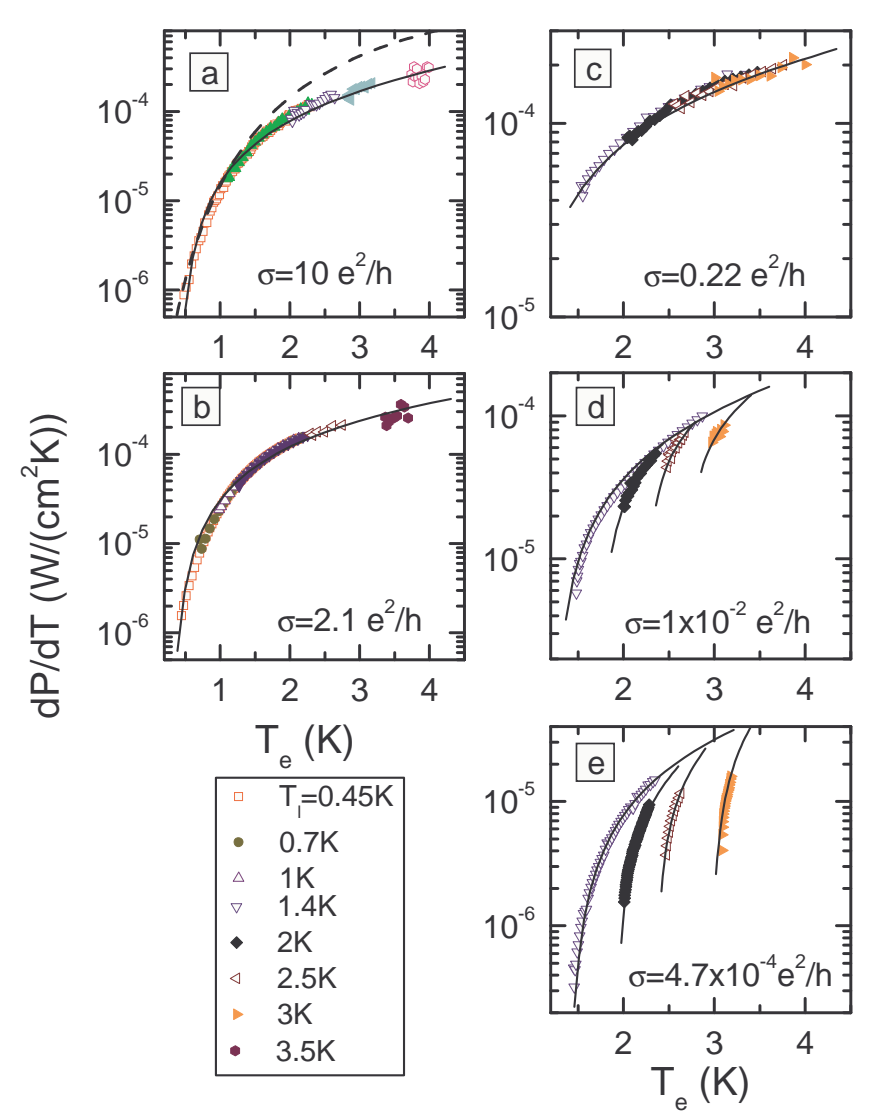

FIG. 2: The relative nonlinearity $\eta$ as a function of electron temperature $T_{e}$ measured at different lattice temperature $T_{l}$ for various conductivities. Dashed line in (a) is calculated according to Ref. 12. Solid lines are provided as a guide for the eye.

at $T=1.5 \mathrm{~K}$.

Figure 1shows the temperature dependence of the conductivity measured in Ohmic regime and power dependences of $\sigma$ for two significantly different conductivity values. The dependences $\sigma(Q)$ were measured at sweeping of the current through the sample from minus $j$ to plus $j$ during several minutes. Within experimental error the $\sigma$-versus- $Q$ plots were identical for both current directions.

At $\sigma>e^{2} / h$ the temperature dependence of the conductivity is close to the logarithmic one [Fig. [1(a)]. Such a temperature dependence is determined by the quantum corrections and was discussed in detail in our previous papers ${ }^{9.13}$ The increase of the conductivity with increasing injected power is result of electron heating and rise of electron temperature from $T_{l}$ to $T_{e}$. For this case, the electron temperature $T_{e}$ at given power $Q$ can be determined by a standard manner ${ }^{14}$ using the experimental dependences $\sigma(T)$ measured at $Q \rightarrow 0$ and $\sigma(Q)$ measured at fixed temperature $T$ as shown in Fig. I(a) by arrows: for each value of $Q, \sigma$ is used as a thermometer giving $T_{e}$ as the temperature $T$ at which the same value of $\sigma$ is measured for $Q \rightarrow 0$.

To obtain the relative nonlinearity we have numerically differentiated the experimental dependences $\sigma(T)$ and $\sigma(Q)$ and found $\eta$ in accordance with Eq. (4). This quantity as a function of electron temperature taken at different lattice temperature is presented in Fig. 2

First we consider the results for highest conductivity $\sigma=10 e^{2} / h$ which unambiguously corresponds to the diffusion regime [Fig. 2(a)]. It is clearly seen that $\eta$ versus- $T_{e}$ data obtained for different lattice temperatures fall on common curve as it has to be in the diffusive regime. This curve is close to theoretical dependence $\partial F\left(T_{e}\right) / \partial T_{e}$ when the interaction with deformation and piezoelectric potential of the acoustic phonons is taken into account 12

Such a data processing was carried out over the whole conductivity range and the results are presented in Figs. 2(b)-(e). It is evident that $\eta$-versus- $T_{e}$ data obtained for different lattice temperatures fall on common curve down to $\sigma=0.22 e^{2} / h$ and only at $\sigma=$ $1 \times 10^{-2} e^{2} / h$ some deviation arises. This deviation becomes drastic at $\sigma=4.7 \times 10^{-4} e^{2} / h$. It points to the fact that one or more specific features of the hopping regime discussed above occur: the conductivity becomes dependent both on the lattice and electron temperature; the electric field leads to the conductivity change not only via electron heating but through the increase of the probability of hops and impact ionization; the approximation of electron temperature fails. In means also that the way of finding of the electron temperature can be incorrect at $\sigma<10^{-2} e^{/} h$, and the quantity $T_{e}$ in Fig. 2 is some effective temperature.

In summary, we have proposed the way how studying the conductivity nonlinearity one can determine the condition when the diffusion regime changes to the hopping one. We have shown that for single quantum well $\mathrm{GaAs} / \mathrm{InGaAs} / \mathrm{GaAs}$ heterostructures the conductivity behaves like diffusive one down to $\sigma \approx(2-3) \times 10^{-2} e^{2} / h$. The conclusion that the low-temperature conductivity of the disordered 2D systems remains diffusive down to $\sigma \ll e^{2} / h$ agrees with that obtained from the studies of the quantum corrections to the conductivity at decreasing of $k_{F} l$ carried out in Ref. 9 .

This work was supported in part by the RFBR through Grants No. 01-02-17003 and No. 03-02-16150, the INTAS through Grant No. 1B290, the Program University of Russia, the CRDF through Grant No. REC-005, and the Russian Ministry of Education through Grant No. A032.9-521.

1 N. F. Mott and W. D. Twose., Adv. Phys. 10107 (1961).

${ }^{2}$ B. I. Shklovski and A. L. Efros, Electronic Properties of 
DopedSemiconductors, Springer, Berlin, 1984.

3 H.W. Jiang, C.E. Jonson, K.L. Wang. Phys. Rev. B 46, 12830 (1992).

${ }^{4}$ H.W. Jiang, C.E. Jonson, K.L. Wang, S.T Hannahs. Phys. Rev. Lett. 71, 1439 (1993).

${ }^{5}$ T. Wang, K.P. Clark, G.F. Spenser, A.M. Mack, W.P. Kirk. Phys. Rev. Lett. 72, 709 (1994).

${ }^{6}$ F.V. Van Keuls, X.L. Hu, H.W. Jiang, A.J. Dahm Phys. Rev. B 56, 1161 (1997).

7 C.H. Lee, Y.H. Chang, Y.W. Suen, H.H. Lin. Phys. Rev. B 58, 10629 (1998).

8 M. E. Gershenson, Yu. B. Khavin, D. Reuter, P. Schafmeister, and A. D. Wieck. Phys. Rev. Lett. 85, 1718 (2000).

9 G. M. Minkov, O. E. Rut, A. V. Germanenko, A. A. Sherstobitov, B. N. Zvonkov, E. A. Uskova, and A. A. Birukov,
Phys. Rev. B 65, 235322 (2002).

10 Estimations show that approximation of electron temperature is valid at $n>10^{14} \mathrm{~m}^{-2} 11$

11 I. L. Drichko, A. M. Dyakonov, V. D. Kagan, A. M. Kreshchuk, T. A. Polyanskaya, I. G. Savel'ev, I. Yu. Smirnov, and A. V. Suslov. Fiz. Tekn. Poluprov. 31, 1357 (1997) [ Semicond. 311170 (1997)].

12 P. J. Price, J.Appl. Phys. 53, 6863 (1982).

13 G. M. Minkov, O. E. Rut, A. V. Germanenko, A. A. Sherstobitov, V. I. Shashkin, O. I. Khrykin, and B. N. Zvonkov, Phys. Rev. B 67, 205306 (2003).

14 R. Fletcher, Y. Feng, C. T. Foxon, and J. J. Harris. Phys. Rev. B 61, 2028 (2000). 